\title{
Effects of Interaural Intensity Difference on the Processing of Interaural Time Difference in the Owl's Nucleus Laminaris
}

\author{
Svenja Viete, José Luis Peña, and Masakazu Konishi \\ Division of Biology, California Institute of Technology, Pasadena, California 91125
}

Interaural time and intensity differences (ITD and IID) are processed independently in the owl's auditory system. This paper examines whether this independence is established in nucleus laminaris (NL), the first site of ITD processing. A plot of discharge rate against time difference (ITD curve) is sinusoidal in NL. The ITDs that produce the peaks are called the most favorable ITDs, and those that produce the troughs are called the least favorable ITDs. IID had little effect on the discharge rates of laminaris neurons for the most and least favorable ITDs. The degree of peak-trough modulation changed slightly with variation in IID. In contrast, IID in tonal stimuli affected the temporal aspect of ITD curves depending on the difference between the stimulus frequency and the neuron's best frequency (BF). For frequencies below BF, IID caused large and systematic shifts in ITD toward the ear in which the sound was louder, whereas for frequencies above BF, IID caused small shifts in ITD toward the opposite ear. IID had little effect on ITD curves taken with BF or broadband noise. These results can be largely accounted for by the effects of frequency and intensity on the timing of impulses at the level of the cochlear nuclei. Thus, the processing of ITD by NL neurons is independent of IID for behaviorally relevant stimuli, because the timing of impulses is insensitive to sound level when the signal is broadband.

Key words: owl; sound localization; nucleus laminaris; interaural time difference; interaural intensity difference; parallel pathways
Parallel processing of information is an important operation in many sensory systems (see, for example, owl's auditory system: Takahashi et al., 1984; monkey visual system: Maunsell et al., 1990; Merigan and Maunsell, 1993; fish electrosensory system: Heiligenberg, 1991). The barn owl's auditory brainstem offers a relatively simple system in which to analyze the relationship between parallel pathways. The owl uses interaural time and intensity differences (ITD and IID) for sound localization (Moiseff, 1989b). The independence of these cues is important for the species, because they code for different spatial coordinate axes, ITD mainly for azimuth and IID mainly for elevation. ITD and IID are processed in separate parallel pathways that start at the level of the cochlear nucleus. The avian auditory system has two anatomically and physiologically distinct cochlear nuclei, nucleus magnocellularis (NM) and nucleus angularis (NA). NM neurons lack dendrites and contain end bulbs of Held, whereas NA neurons have dendrites and bouton-type synapses (Parks, 1981; Jhaveri and Morest, 1982; Takahashi and Konishi, 1988a; Carr and Boudreau, 1993). NM neurons show phase locking, whereas NA neurons do not except for very low frequencies (Sachs and Sinnott, 1978; Sullivan and Konishi, 1984a; Konishi et al., 1985; Warchol and Dallos, 1990). The time- and intensity-processing pathways converge in the inferior colliculus, where neurons selective for

Received Sept. 19, 1996; revised Dec. 4, 1996; accepted Dec. 16, 1996.

This work was supported by National Institute of Neurological Disorders and Stroke Grant DC-00134 and postdoctoral fellowships from the Deutsche Forschungsgemeinschaft (S.V.) and the Pew Latin American Fellows Program (J.L.P.) We thank Jamie Mazer and Chris Malek for assistance with computer programming, Yehuda Albeck for advise on data analysis, and Yehuda Albeck, Ben Arthur, Catherine Carr, Roian Egnor, Jamie Mazer, Walter Metzner, Terry Takahashi, Larry

Proctor, and Marc Schmidt for reading early versions of this manuscript.

Correspondence should be addressed to Masakazu Konishi, Division of Biology 216-76, California Institute of Technology, Pasadena, CA 91125.

Copyright (C) 1997 Society for Neuroscience $0270-6474 / 97 / 171815-10 \$ 05.00 / 0$ combinations of ITD and IID are found. Partial inactivation of NM affects only the ITD tuning of collicular neurons, whereas the same treatment of NA affects only the IID tuning of the same neurons (Takahashi et al., 1984). These findings were the bases for the theory of independent processing of ITD and IID.

It is not known, however, whether the first neurons in the time pathway that process ITD are insensitive to IID. ITDs are detected by a neuronal circuit consisting of axons from NM, serving as delay lines, and neurons of the nucleus laminaris (NL), serving as coincidence detectors (Sullivan and Konishi, 1984b; Young and Rubel, 1986; Carr and Konishi, 1990; Overholt et al., 1992; Carr, 1993; Joseph and Hyson, 1993). NL is thought to be homologous or similar to the medial superior olivary nucleus of mammals (MSO). NL and MSO neurons resemble each other except that the owl's NL neurons can operate at much higher frequencies. Moreover, in barn owls, ITD and IID are not processed in different frequency ranges as in mammals (Goldberg and Brown, 1969; Yin and Chan, 1990). Detection of ITD and IID in the same high-frequency range that the barn owl uses for sound localization would pose problems if the time-processing pathway were sensitive to large IIDs $(20-25 \mathrm{~dB})$ that occur with high frequencies under natural conditions (Moiseff, 1989a,b; Brainard et al., 1992). The present paper examines whether or how normally occurring IIDs affect the response of NL neurons to ITD.

\section{MATERIALS AND METHODS}

\section{Surgery}

We used 12 adult barn owls (Tyto alba) of both sexes. These animals also provided data for other studies of the NL (Peña et al., 1996). Owls were anesthetized by intramuscular injection of ketamine hydrochloride $(25$ $\mathrm{mg} / \mathrm{kg}$; Ketaset) and Diazepam (1.3 mg/kg; Western Medical Supply) for placement of a small stainless-steel plate on the skull that held the head during subsequent experiments. Recording sessions began 5-7 d after this initial operation. Anesthesia was induced and maintained as described 
above. We opened a small hole on the skull area overlying NL and cut a slit in the dura mater for electrode insertion. At the end of a recording session, lasting 4-5 hr, we covered the craniotomy with a plastic sheet and dental cement and closed the skin incision. We applied an antibiotic ointment (Bacitracin Zinc-Neomycin Sulfate-Polymyxin B Sulfate, E. Fougera and Co.) to the skin wounds. Owls were returned to their individual cages and monitored for their recovery. Depending on the owls' weight and recovery, experiments were repeated every 7-10 d for a period of several weeks.

\section{Acoustic stimuli}

Custom software was available for the synthesis of sound stimuli, data collection, and analysis (Mazer, 1995). Acoustic stimuli were synthesized on a computer (Sparc/IPX, Sun Microsystems) and presented by a digital signal processor equipped with a 16-bit, $48 \mathrm{kHz}$ data acquisition subsystem (S56x + Proport, Berkeley Camera Engineering). Tonal and broadband stimuli (100 msec in duration, $5 \mathrm{msec}$ linear rise/fall time) were presented once per second. ITD was varied in steps of either one-tenth of the period for tonal stimuli or $30 \mu \mathrm{sec}$ for noise stimuli. Stimulus intensities could be varied in steps of $1 \mathrm{~dB}$ with a pair of digitally controlled attenuators (PA4, Tucker Davis Technologies).

All experiments took place in a double-walled sound-attenuating chamber. Acoustic stimuli were delivered by earphones (Sony MDRE535) attached to a metal delivery tube $(3 \mathrm{~cm}$ long, $4 \mathrm{~mm}$ inner diameter). A small microphone (ED-1939, Knowles Electronics) with a probe tube was used to measure sound intensity at the end of the delivery tube. The gaps between the earphone assembly and the ear canal were filled with silicone impression material (Gold Velvet, JKR Laboratories). Sound intensities were measured in the ear canal at a distance of $\sim 1 \mathrm{~mm}$ from the ear drum using a $12.5 \mathrm{~mm} \mathrm{~B} \mathrm{\&} \mathrm{K} \mathrm{microphone} \mathrm{with} \mathrm{a} \mathrm{calibrated}$ probe tube ( $1 \mathrm{~mm}$ outer diameter, $5 \mathrm{~mm}$ length). This tube was inserted through a hole made in the squamosal bone which forms the roof of a cavity over the ear drum. Simultaneous measurement of sound with both the B \& K and the Knowles microphones made it possible to translate the voltage output of the Knowles into sound intensity in dB SPL. The Knowles microphones were then used to calibrate the earphone assemblies at the beginning of each experimental session.

The calibration data contained the amplitudes and phase angles measured in steps of $100 \mathrm{~Hz}$. Differences in amplitude between the two earphones could usually be reduced by repositioning the earphones. For phase differences, appropriate corrections were made in the affected data. Irregularities in the frequency response of each earphone were automatically smoothed by the computer from 4 to $9 \mathrm{kHz}$. The study of neuronal responses was restricted to cells tuned to frequencies above $4 \mathrm{kHz}$, because lower-frequency sounds pass from one middle ear to the other through the interaural canal (Moiseff and Konishi, 1981). This cross-talk can confound the study of neurons tuned to lower frequencies.

\section{Data collection}

We obtained all data by a "loose patch" method that permitted well isolated and stable extracellular recordings. This is an important technical advance in the study of NL, because isolation of single neurons is very difficult to obtain, presumably because of the sparsely distributed neuronal somata and the large field potentials present in this area. Even if neurons are isolated, they are difficult to maintain mostly because of brain pulsation. Similar difficulties have been encountered in most of the studies in both NL and the medial superior olivary nucleus (MSO) of mammals (for relevant references, see Peña et al., 1996). In the present study, the number of neurons that could be obtained in each experimental session was still small, but they could be maintained for 1-2 hr, during which an extensive test protocol could be carried out.

We prepared patch electrodes from $1.0 \mathrm{~mm}$ borosilicate glass (World Precision Instruments) using a micropipette puller (Sutter Instruments $\mathrm{P}-87$ ). Electrodes were filled with a patch solution (in mM: K-gluconate 100, EGTA 10, HEPES 40, $\mathrm{MgCl}_{2}$ 5, Na-ATP 2.2, Na-GTP 0.3). Electrode impedance ranged from 4 to $10 \mathrm{M} \Omega$. Broadband noise bursts with ITD and IID set to zero were used as a search stimulus. NL was located stereotaxically and by its physiological response properties. At $1.5-2.5$ $\mathrm{mm}$ posterior to the interaural axis and $1.5-2.0 \mathrm{~mm}$ from the midline, it is usually $8-9 \mathrm{~mm}$ below the surface of the brain. NL can also be recognized by neurophonic potentials that closely resemble the stimulus waveform. In the owl's brainstem, NM and NL are the only nuclei that produce neurophonic potentials, presumably because their neurons phase-lock to the stimulus. NL neurophonic potentials show ITD tuning, whereas those in NM do not.
Electrodes were advanced with a microdrive (Motion Controller, Model PMC 100) in steps of $100 \mu \mathrm{m}$ until NL was reached. The size of the steps was reduced to $3-5 \mu \mathrm{m}$ to search for and isolate single neurons in NL. During this process, we applied a positive pressure to the tip of the electrode to prevent clogging that could be easily detected by changes in impedance. When small sound-evoked impulses became recognizable, we carried out a simple test for discriminating between monaural and binaural responses. Application of a negative pressure at the tip of the electrode often led to good isolation of the spiking cell even without achieving a high-resistance seal. Once this degree of isolation was obtained, the electrode was seldom dislodged from the cell. We have been able to maintain cells for $>2 \mathrm{hr}$ without any sign of diminishing impulse amplitude. The health of the cells gradually deteriorates in conventional whole-cell patch recording, because they lose ions and other molecules by diffusion into the electrode. This problem did not occur with our method, because the electrode did not appear to break the cell membrane; it worked as a suction electrode. Neural signals were recorded with an Axoclamp-2A amplifier (Axon Instruments) in the conventional currentclamp bridge mode. The signal was amplified further, filtered (0.3-10 $\mathrm{kHz}$ ), and discriminated with a custom-made voltage level detector. Both the Axoclamp-2A and level detector outputs were digitized and stored by the computer at a sampling rate of 24 or $48 \mathrm{kHz}$.

The same loose-patch method was used to record from NM axons. Axons coming from both the ipsilateral and the contralateral NM could be easily recorded as they coursed across NL (cf. Carr and Konishi, 1990).

\section{Data analysis}

Impulse numbers obtained for specific values of stimulus parameters such as frequency, ITD, and IID constitute raw data in the present study. Responses are seldom sampled for every parameter value. Therefore, the relationship between a given parameter and impulse number must be derived from samples obtained for selected parameter values. The methods of derivation must be objective and appropriate for the purpose of the experiment. We have developed automatic methods to measure neuronal threshold, dynamic range, and asymptotic level on response curves closely fitted to the distributions of raw data (Peña et al., 1996).

Mean interaural phase. In the present work, we measured the mean interaural phase (MIP) by a technique that differs from the method of vector summation (Goldberg and Brown, 1969; Batschelet, 1981). We obtained MIPs from cosine curves to which data points were fitted. This procedure is justifiable, because the mean impulse rates of an NL neuron to different ITDs fit better to a cosine function than to a curve obtained by their linear interpolation (Fig. 1). The data for the fitting were mean impulse rates and their SDs obtained by 15 repetitions of each ITD. The fitting equation is ITD curve (in impulses/sec) $=a_{1}+$ $a_{2} \exp \left(-a_{5} \theta^{2}\right) \cos (\theta)$, where $\theta=a_{3}$ ITD $+a_{4}, a_{1}$ is the average amplitude of ITD curve, $a_{2}$ is the maximal response level, $a_{3}$ is the period of the cosine function, and $a_{4}$ is the mean interaural phase. $a_{5}$, the definition of which is given below, was set to zero in fitting data obtained with tones. We used the Levenberg-Marquardt method to fit the data to the above equation (Press et al., 1988). This technique automatically fits mean impulse rates to the equation by minimizing the differences between them. For ITD curves obtained with noise stimuli, we used the distance between the peaks to estimate the period of the cosine function $a_{3}$ and its phase $a_{4}$, the mean response to estimate $a_{1}$, and the maximal response to estimate $a_{2}$. The best fit for the noise results was obtained when the peaks of the cosine curve declined with ITD in a Gaussian manner as shown by the term $a_{2} \exp \left(-a_{5} \theta^{2}\right)$ in the equation, where $a_{5}$ determines the rate of decline. An initial value of $1.0 /(500$ $\mu \mathrm{sec})^{2}$ was always used to estimate $a_{5}$.

We used a $\chi^{2}$ method to determine the goodness of fit, i.e., $\chi^{2}=$ $\Sigma\left(y_{i}-x_{i}\right)^{2} / \sigma_{i^{2}}$, where $i$ is an index for a sampled ITD, $y_{i}$ is the mean spike rate for the $i$ th ITD, $x_{i}$ is the impulse rate obtained by the cosine fitting for the $i$ th ITD, and $\sigma_{i}$ is the standard deviation of the $i$ th mean (Press et al., 1988). The criterion for a moderately good fit is $\chi^{2} \approx$ the degree of freedom, which is the number of sampled ITDs minus the number of fitting parameters. We obtained much better fits (smaller $\chi^{2}$ values) than that defined by the above criterion. All of the ITD data we used for computation of MIP satisfied our more stringent criterion (see Fig. 1). Also, most MIP values obtained by the vector summation method did not differ from those obtained by the cosine-fitting method.

Mean phase and vector strength. Both NM and NL neurons lock to the phase of tonal stimuli. We measured the preferred phase and the degree of phase-locking using the methods of vector summation (Goldberg and 


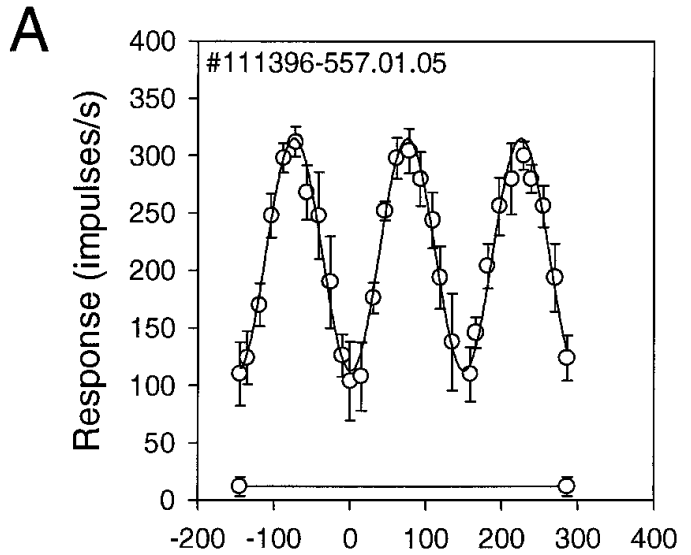

B

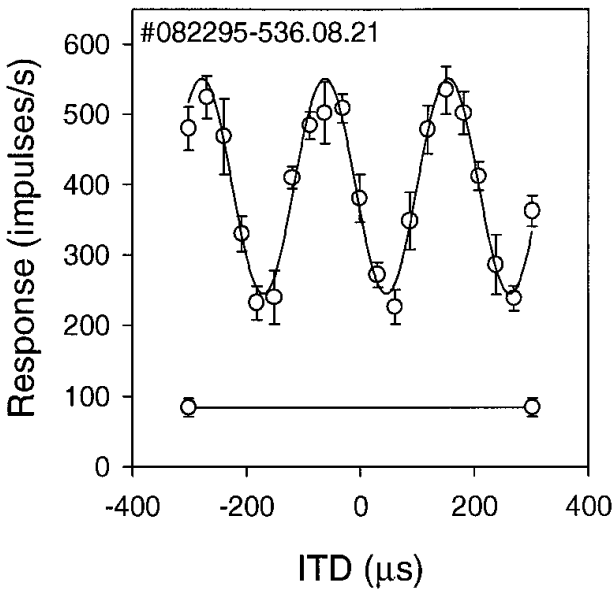

Figure 1. Fitting ITD responses to cosine function. Circles show the mean discharge rates, and vertical bars show the SEMs. $A$, A very good fit with $\chi^{2}=6.9(\mathrm{df}=25)$ for ITD data obtained with a tone. $B$, A poor fit with $\chi^{2}=13.1(\mathrm{df}=16)$ for ITD data obtained with noise. Horizontal lines indicate spontaneous discharge level.

Brown, 1969). A neuron's preferred phase is represented by the direction of the mean vector ( $\mathrm{MP}=$ mean phase), which is given by the following equation:

$$
\begin{aligned}
\mathrm{MP} & =\arctan \left(\Sigma y_{i} / \sum x_{i}\right) \\
x_{i} & =R_{i} \cos \beta \\
y_{i} & =R_{i} \sin \beta,
\end{aligned}
$$

where the mean impulse number $\left(R_{i}\right)$ at the $i$ th phase $(\beta)$ bin defines a vector with two components $\left(x_{i}\right.$ and $\left.y_{i}\right)$.

Vector strength (VS), which is the length of the mean vector, represents the degree of phase-locking and is given by:

$$
\mathrm{VS}=\sqrt{\left(\left(\sum x_{i}\right)^{2}+\left(\Sigma y_{i}\right)^{2}\right) / \Sigma R_{i}} .
$$

VS varies from 0 , indicating no phase-locking, to 1.0, indicating all impulses occurring in one bin, which was $20 \mu \mathrm{sec}$.

Sharpness of ITD tuning. We adopted the same concept of vector strength to measure the degree of peak-trough modulation in ITD curves by replacing "phase" by "interaural phase difference" in the VS equation. $\mathrm{VS}_{\text {itd }}$ varies from 0 , indicating no ITD sensitivity, to 1.0, indicating all impulses occurring in one bin, which was $1 / 10$ of the period of the ITD curve. We measured $\mathrm{VS}_{\text {itd }}$ only in tests involving tonal stimuli. The main weakness of this method is its sensitivity to the average amplitude of ITD curves. When the average amplitude is large, $\mathrm{VS}_{\text {itd }}$ becomes small, even if the peak-trough difference remains the same.

Summation ratio. The relationship between binaural and monaural responses was represented by the summation ratio (SR) (Goldberg and Brown, 1969):

$$
\mathrm{SR}=R_{\mathrm{b}}-R_{\text {spont }} /\left(\left(R_{\mathrm{i}}-R_{\text {spont }}\right)+\left(R_{\mathrm{c}}-R_{\text {spont }}\right)\right),
$$

where $R_{\mathrm{b}}$ is the response to binaural stimulation, $R_{\mathrm{i}}$ and $R_{\mathrm{c}}$ are the responses to monaural stimulation at the ipsilateral and the contralateral ears, respectively, and $R_{\text {spont }}$ is the spontaneous discharge. A value of 1 indicates linear summation, whereas values less than 1 and greater than 1 indicate "facilitation" and "disfacilitation," respectively (Goldberg and Brown, 1969).

\section{Anatomy}

The positions of recording electrodes were marked with Neurobiotin $(2 \%$ in patch solution) in the last recording sessions in some of the animals. After tracer injection, the owls were overdosed with sodium pentobarbital (Nembutal, Abbott Laboratories) and perfused first with $0.9 \%$ saline in

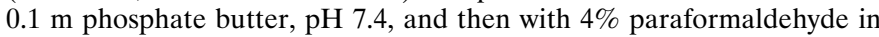
$0.1 \mathrm{M}$ phosphate buffer. Brains were blocked in the plane of electrode penetration, removed from the skull, and placed in $30 \%$ sucrose until they sank. They were then cut into $30 \mu \mathrm{m}$ sections with a freezing microtome, rinsed repeatedly in buffer, prebleached with $0.5 \% \mathrm{H}_{2} \mathrm{O}_{2}$ in phosphate buffer for $10 \mathrm{~min}$, and incubated in buffered $\mathrm{ABC}$ reagent (Vector Laboratories). After multiple rinses, the sections were reacted in a buffered solution containing $0.1 \%$ diaminobenzidine (Sigma) and $0.01 \%$ nickel ammonium sulfate for $15 \mathrm{~min}$ and then incubated with $3 \% \mathrm{H}_{2} \mathrm{O}_{2}$ for $5-15 \mathrm{~min}$. Sections were mounted onto gelatin-coated slides and counterstained with cresyl violet.

\section{RESULTS}

The effects of variation in IID on ITD tuning curves were studied in a total of $30 \mathrm{NL}$ neurons. ITD curves were obtained for different IIDs, whereas the average binaural intensity $(\mathrm{ABI}=$ ipsi + contra intensities in dB SPL divided by 2 ) was maintained constant at the midpoint of the dynamic range of each neuron. The mean dynamic range of $23 \mathrm{NL}$ neurons was $21.0 \pm 12.5 \mathrm{~dB}$ for the most favorable ITD and $26.3 \pm 11.8 \mathrm{~dB}$ for the least favorable ITD (Peña et al., 1996). The numbers of neurons used for each set of experiments are given in each of the following sections.

\section{Discharge rate and summation ratio}

Discharge rates for the most and least favorable ITDs were measured in 12 neurons for their best frequencies (BFs) while IID was varied. Both rates changed little within the $30 \mathrm{~dB}$ range of IIDs tested (Fig. 2). Summation ratios ( $\mathrm{SR}=$ binaural response rate divided by the sum of monaural response rates after subtracting spontaneous rate) were calculated for the most and least favorable ITDs in 10 neurons in which a complete set of relevant data could be obtained. The stimuli were tones of the neurons' BFs. The SR changed little with IID except for extreme values of IID tested $( \pm 30 \mathrm{~dB})$, where they appeared to increase from their normal values of 0.5 (for least favorable) and 1.5 (most favorable) (Peña et al., 1996) (Fig. 3).

\section{The sharpness of ITD tuning}

When the discharge rate of an NL neuron varies greatly between the most and least favorable ITDs, the peaks of its ITD curve appear pointed and the neuron is said to be sharply tuned to the most favorable ITD. We examined whether IID affects the sharpness of ITD tuning in 27 neurons with tonal stimuli. We used vector strength (VS) as a measure of tuning sharpness, because it measures the concentration of impulses at different ITDs. If all ITDs were equally effective in eliciting impulses from a neuron, its vector strength would be 0 . Likewise, if only one ITD elicited impulses, the vector strength would be 1 . The results of this test showed that vector strength appeared to decline slightly as IIDs diverged from zero, i.e., for either the ipsilateral or the contralat- 

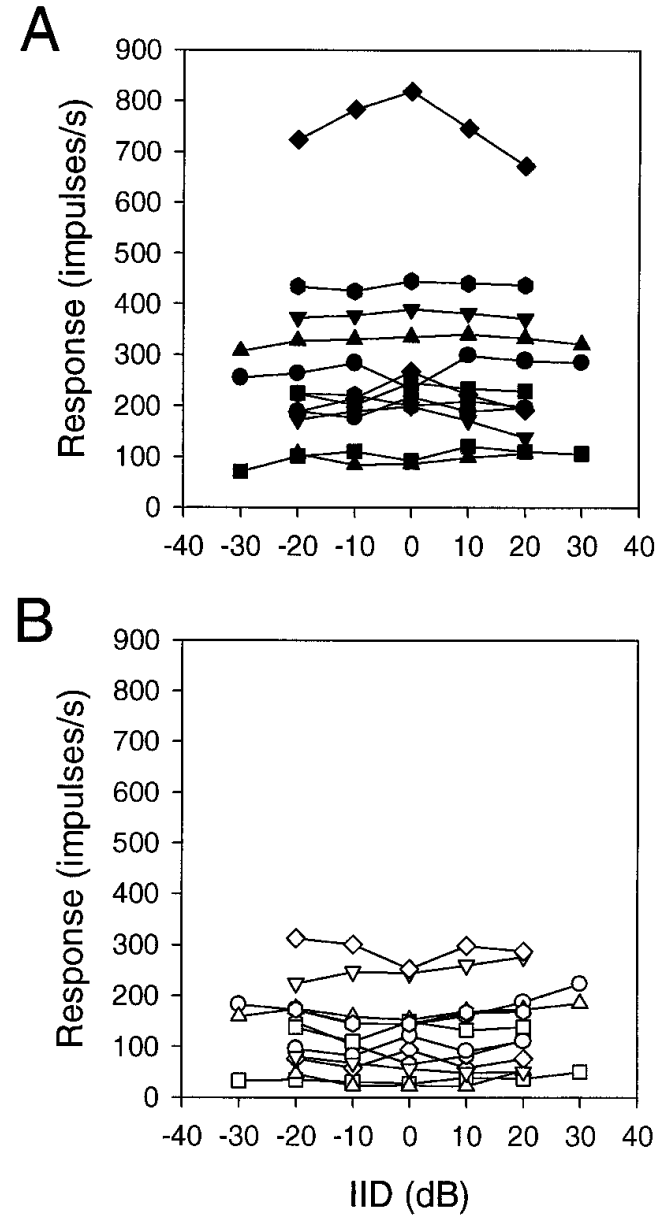

Figure 2. Effects of IID on binaural discharge rates. $A$, Discharge rate for the most favorable ITD. $B$, Discharge rate for the least favorable ITD. Each line connecting the same symbols shows results from a single neuron. Symbols may be used more than once.

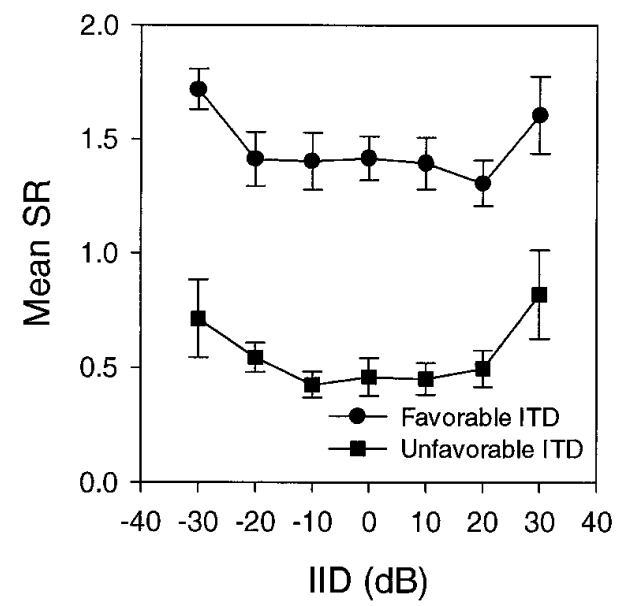

Figure 3. Effect of interaural intensity difference on summation ratio. The mean summation ratios for 10 neurons are plotted against IID. Values for most favorable and least favorable ITDs are plotted separately. Only the differences in these values between IID $=0$ and IID $= \pm 30$ are statistically significant.
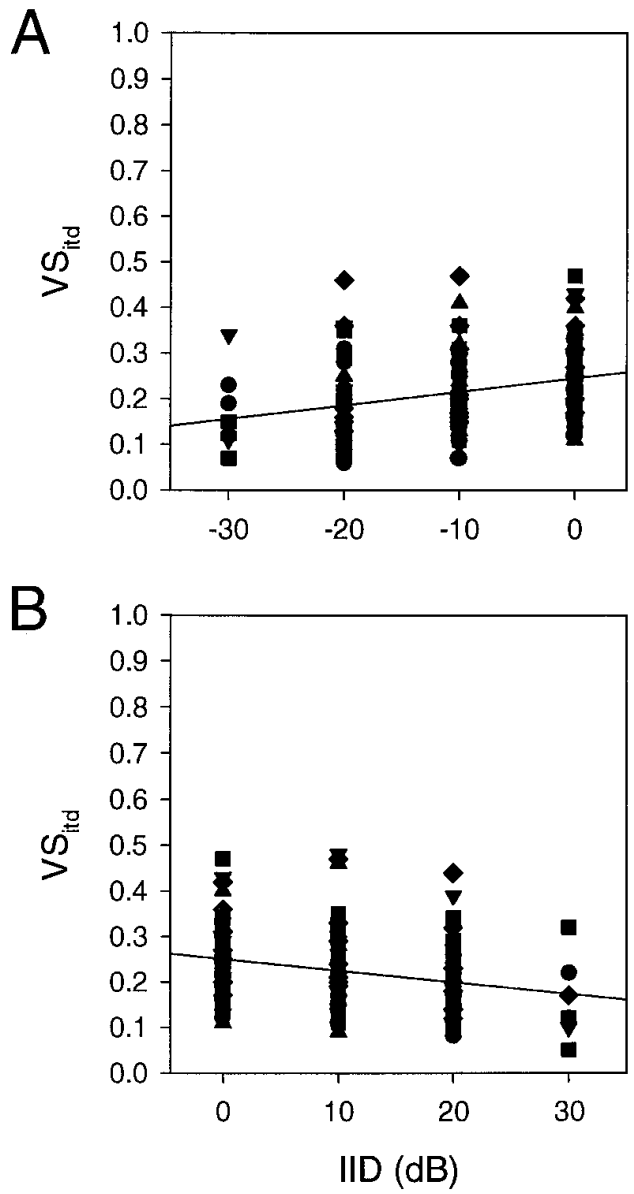

Figure 4. Effects of interaural intensity difference on vector strength $\left(V S_{\text {itd }}\right)$ of ITD curves. $V S_{\text {itd }}$ here provides a measure of distribution of impulses to different ITDs during one period of ITD curves. Larger $V S_{\text {itd }}$ values indicate larger modulation of discharge rates, provided that the level of spontaneous discharge remains constant. Each neuron was tested with the whole range of IID $(-30$ to $+30 \mathrm{~dB})$, but the results for different directions of IID changes are shown separately. $A$, Sound intensity was greater either in the contralateral ear $(B)$ or in the ipsilateral ear. ABI was kept constant in all tests. Although the coefficients of correlation are small for both regression lines, they are statistically significant.

eral side being louder (Fig. 4). A regression analysis of the trend from 0 IID to either +30 or -30 IID showed that these linear correlations were statistically significant. The regression line in Figure $4 A$ had a slope value of $0.0030 \mathrm{VS}_{\text {itd }} / \mathrm{dB}$, and that in Figure $4 B$ had a slope value of $0.0026 \mathrm{VS}_{\mathrm{itd}} / \mathrm{dB}$. The correlation coefficient for the former was $0.317(p=0.00035)$, and that for the latter was $-0.25(p=0.001)$, where $p$ is the probability of observing these coefficients by chance.

\section{The degree of phase locking}

NL neurons phase-lock to the stimulus tone. The degree of phase locking as measured in terms of vector strength varies with ITD (Goldberg and Brown, 1969; Peña et al., 1996). We examined whether IID affected vector strength during the presentation of the most favorable ITD. The results showed that IID had little effect on vector strength irrespective of the stimulus frequency for which we used frequencies equal to $\mathrm{BF}$, below $\mathrm{BF}$, and above $\mathrm{BF}$ (data not shown). 


\section{Mean interaural phase \\ Stimulation with tones}

In this section, we study the effects of IID on the temporal aspect of ITD curves. The question is whether a neuron's most favorable ITD changes when sound intensity in one ear is greater than in the other ear. If the most favorable ITD changes, how it changes is also an important question. The most favorable ITD can shift toward either one or the other ear. For example, the most favorable ITD of a neuron being $+50 \mu \mathrm{sec}$ means that the neuron responds best when the signal in the right ear leads that in the left ear by $50 \mu \mathrm{sec}$. A change of $+20 \mu \mathrm{sec}$ in the ITD as a result of an increase in sound intensity in the right ear is said to be "a shift of $20 \mu \mathrm{sec}$ toward the right ear." Of course, such a shift is not just in the most favorable ITD but in the entire ITD curve. For the purpose of this section, we use mean interaural phase (MIP) as a measure of the most favorable ITD.

ITD tuning curves showed shifts in response to varying IIDs, the magnitude and direction of which depended on the difference between the neuron's BF and the stimulus frequency. The frequency-dependent shift of MIP is exemplified for one representative NL neuron in Figure 5. When stimulated with a frequency $250 \mathrm{~Hz}$ below its BF $(6250 \mathrm{~Hz})$, its MIP shifted steadily to more positive values (contralateral side leading) as IID increased (contralateral side louder). Stimulation at the neuron's BF (6500 $\mathrm{Hz}$ ) resulted in steady but less dramatic MIP shifts with increasing IID. IID had little effect on MIP when the stimulus frequency $(7250 \mathrm{~Hz}$ ) was $750 \mathrm{~Hz}$ above BF.

Quantitative data on MIP shifts were obtained for tonal stimuli at the neuron's BF, frequencies above and below BF, as well as for broadband noise. Fourteen neurons were tested at frequencies $150 \mathrm{~Hz}$ below BF (Fig. 6A), 11 neurons tested at BF (Fig. 6B), and 18 neurons tested at frequencies at least $150 \mathrm{~Hz}$ above $\mathrm{BF}$ (Fig. 6C). Stimulation at BF resulted in small MIP shifts the direction of which differed between neurons. In addition, the magnitude of MIP shifts varied linearly with IID in 8 of 11 neurons and did not vary in 3 neurons. The linear shifts tended to be toward the louder ear (Fig. 6B). The magnitude of MIP shifts for frequencies above the neurons' BFs increased as a linear function of IID in a majority of the neurons. However, the direction of MIP shifts varied between neurons; 6 neurons changed their MIPs toward the louder ear, and 11 neurons changed their MIPs toward the quieter ear. Stimulation with frequencies lower than BF caused larger and more consistent MIP shifts toward the louder ear than with any other stimulus. The magnitude of these shifts varied linearly with IID in all neurons tested.

To summarize the observed MIP shifts, we plotted the slopes of MIP shifts with IID against frequency (Fig. 7). Each point represents the slope value obtained from the regression line through each set of data points shown in Figure 6. These slope values were obtained from the neurons that had regression coefficients $>0.8$ and $p<0.05$. This selective procedure excludes the neurons showing no correlation. The direction of MIP shifts is correlated with the difference between BF and the stimulus frequency (Fig. $7 B$ ), but there is no apparent correlation with either BF (Fig. 7A) or the stimulus frequency itself (data not shown). When the stimulus frequency was the neuron's BF, its MIP remained essentially the same. The MIP shifted significantly toward the louder side for frequencies below BF, whereas it shifted slightly toward the softer side for frequencies above BF. Note that for the same
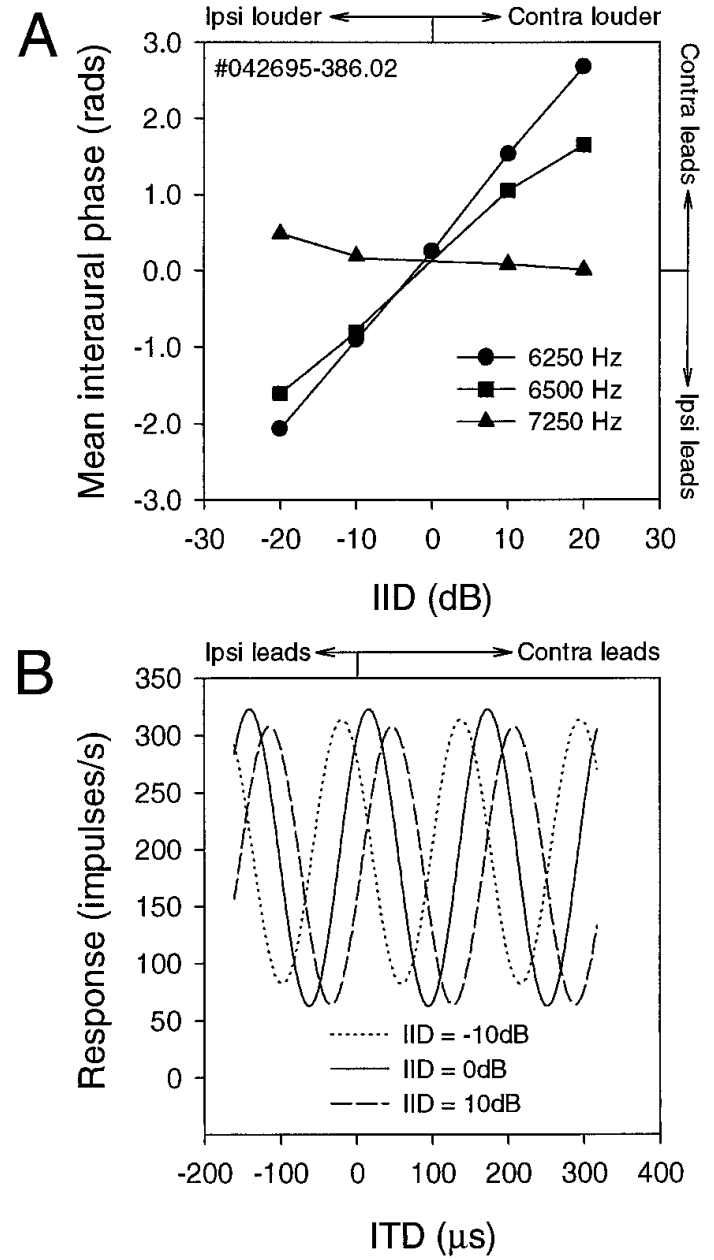

Figure 5. Effects of interaural intensity difference on mean interaural phase with tonal stimuli. $A$, This NL neuron was stimulated with three different frequencies: $6250 \mathrm{~Hz}$ (i.e., below BF, circles), $6500 \mathrm{~Hz}$ (i.e., at BF, squares), and $7250 \mathrm{~Hz}$ (i.e., above BF, triangles). When stimulated with a frequency $250 \mathrm{~Hz}$ below its BF, the MIP shifted steadily toward the louder ear as IID increased. Stimulation with the neuron's BF also resulted in a steady but less drastic shift in the same direction. The MIP appeared to remain almost unchanged for a frequency $750 \mathrm{~Hz}$ above BF. $B$, Three different cosine-fitted ITD curves used to make the $6250 \mathrm{~Hz}$ line in $A$, showing the ITD curve shifts with IID.

amount of departure from BF and the same IID, shifts were greater for lower frequencies than for higher frequencies.

\section{Stimulation with noise}

We tested the effects of IID on ITD processing with broadband signals because of their ethological significance. Ten neurons were stimulated with noise, and ITD data were obtained for different IIDs with ABI kept constant. The MIP tended to shift slightly with increasing IID toward the louder ear in most of the cases, although the magnitude of the shifts appeared to differ between neurons (Fig. 8). For the neurons that had regression coefficients $>0.8$ and $p<0.05$, the average rate of shift was $0.71 \pm 0.22$ $\mu \mathrm{sec} / \mathrm{dB}$.

\section{Responses of afferent axons to IID}

The response of NL neurons to variation in sound intensity may be accounted for by that of their afferent fibers. To test this idea, we studied the effect of both intensity and frequency on the response of NM axons inside the boundaries of NL (Fig. 9). We 


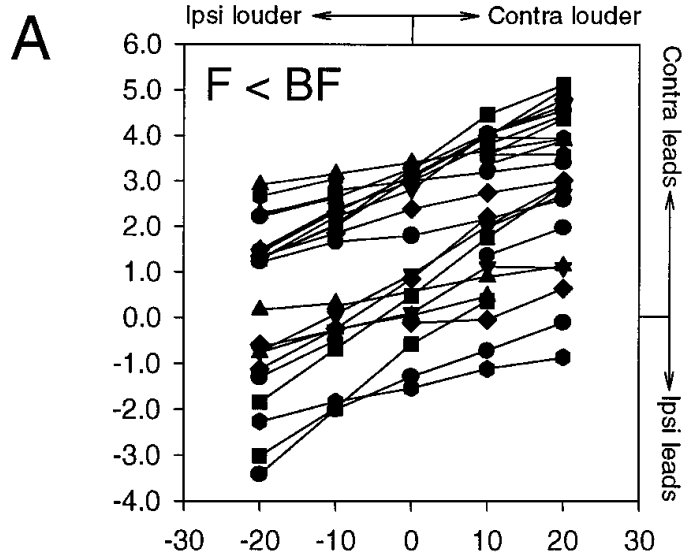

B
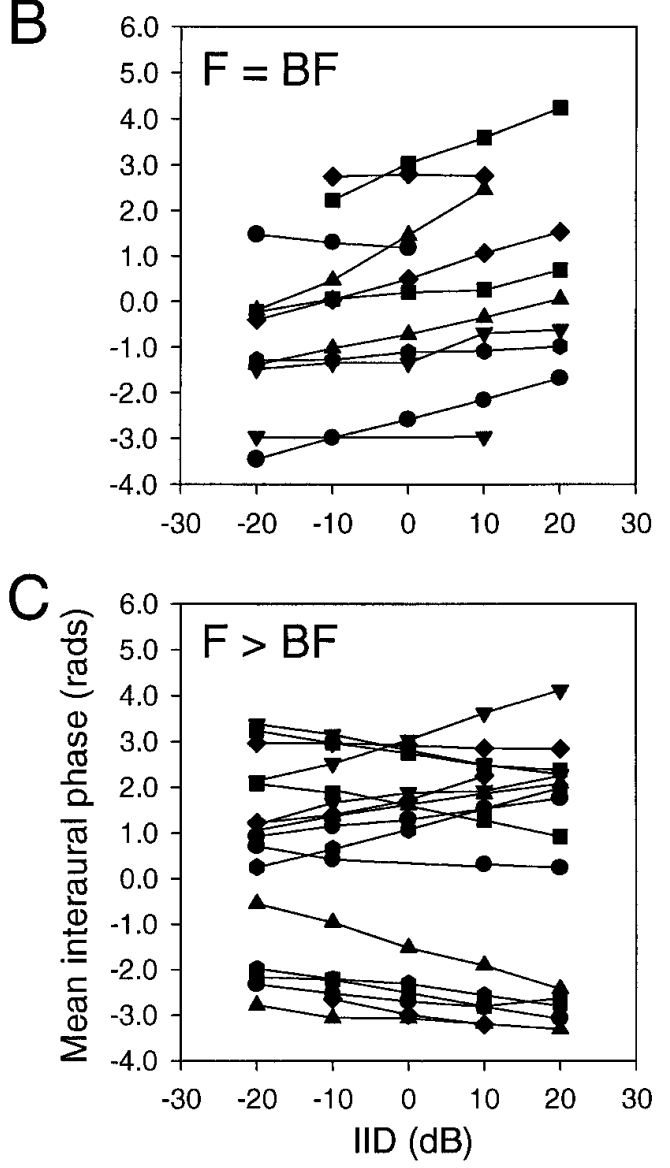

Figure 6. Effects of interaural intensity difference and frequency on mean interaural phase. $A$, Stimulation with frequencies below BF $(n=14)$. The MIP shifted toward the louder ear. $B$, Stimulation with frequencies at or near BF $(n=11)$. The shift of MIP was in the same direction as, yet smaller than, $A$. $C$, Stimulation with frequencies above $\mathrm{BF}(n=18)$. The direction of MIP shifts varied. Five neurons displayed a shift in the MIP toward the louder ear, nine neurons showed MIP shifts in the opposite direction, and four neurons showed no change in MIP.

made period histograms to calculate the mean phase (MP) in 21 neurons for different stimulus frequencies and intensities. Only fibers with vector strengths $>0.2$ were included in the computation of MP. Shifts in MP were not correlated with the neurons' BF (Fig. 9A). Figure $9 B$ shows the relationship between stimulus frequencies and phase shifts for frequencies above, below, and equal to the neurons' BFs. When the stimulus frequency was the
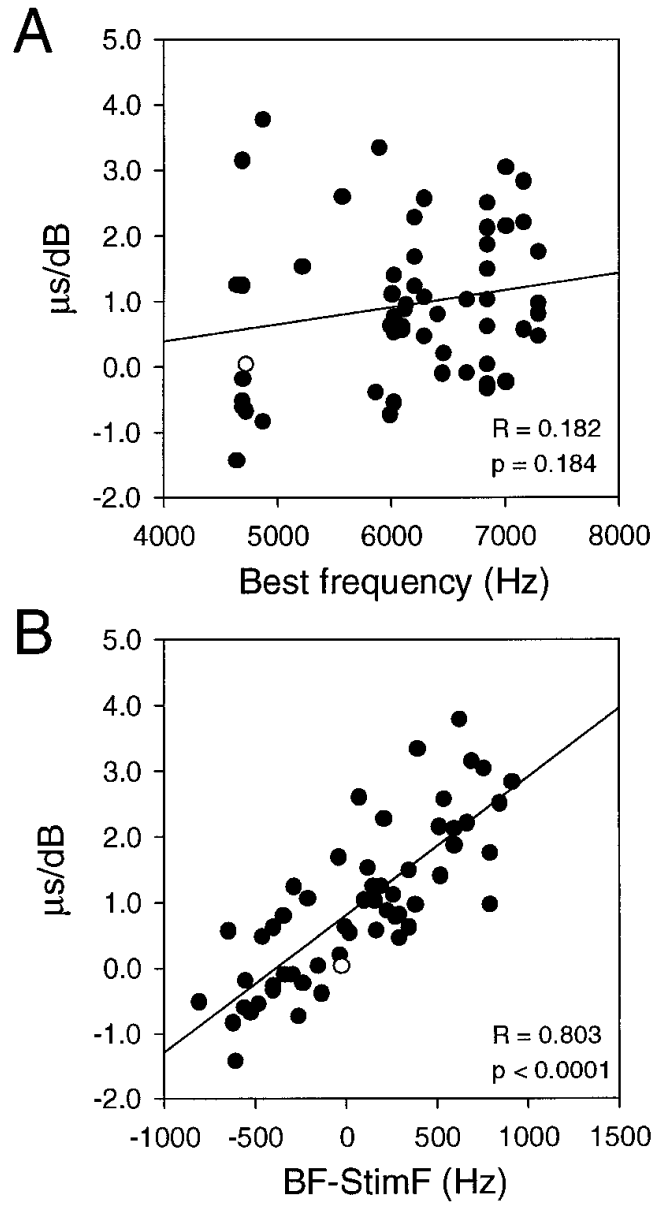

Figure 7. Summary of frequency-dependent shifts of MIP. The rates of change of MIP with IID are plotted against frequency. Each point represents the slope of the regression line through each set of data points shown in Figure 6. There is no apparent correlation with $\mathrm{BF}(A)$. The direction of MIP shifts is correlated with the difference between BF and the stimulating frequency $(B)$. Here, -500 and -1000 on the $B F-S t i m F$ axis indicate frequencies higher than BF. The MIP increases (contralateral side leading) slightly with larger IIDs (contralateral side louder) for frequencies equal to BF. It increases drastically for frequencies below BF. The MIP decreases (ipsilateral side leading) slightly for frequencies higher than BF. Note that the frequency-dependent shift of MIP for different IIDs is not symmetric around zero. Filled symbols indicate neurons in which the MIP shifted linearly with IID with regression coefficients $>0.8$ and $p<0.05$. Open symbols indicate neurons that did not meet these criteria.

same as the neurons' BFs, the MP changed little with variation in sound intensity in a majority of the neurons. The MP increased linearly with sound intensity when the stimulus frequency was below BF, whereas the MP decreased with sound intensity when the stimulus frequency was above BF (Fig. 9B). However, for the same amount of departure from BF and the same sound intensity, shifts were greater for lower frequencies than for higher frequencies (Fig. 9B).

\section{Insensitivity of NM neurons to contralateral stimulation}

Although NM neurons receive only ipsilateral input, variation in sound intensity in the opposite ear may affect their responses, because the two sides might be equalized by negative feedback mechanisms via the superior olivary nucleus (SO), as Lachica et al. (1994) suggested. According to these authors, SO receives bilateral input from NA and NL and sends inhibitory output 


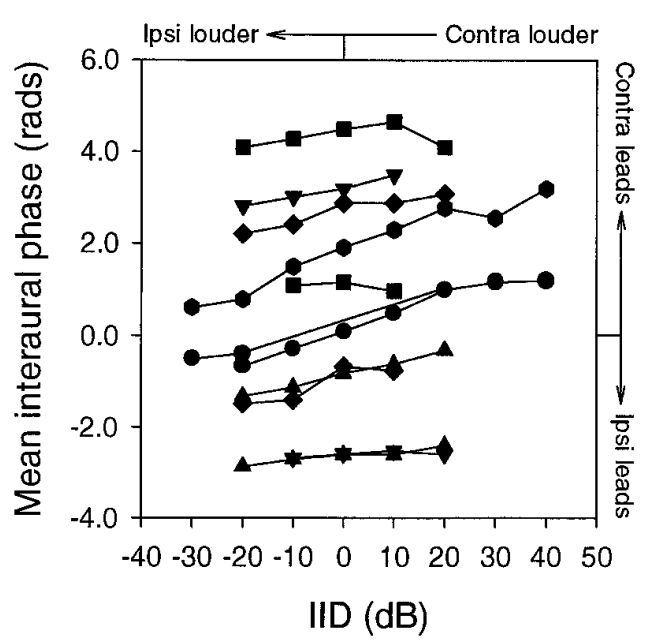

Figure 8. Effects of interaural intensity difference on mean interaural phase with noise. When the stimulus was a broadband noise, the magnitude of shifts in the MIP tended to be small. The mean MIP shift per dB IID of $10 \mathrm{NL}$ neurons is $0.71 \pm 0.22 \mu \mathrm{sec} / \mathrm{dB}$.

bilaterally to these nuclei and NM. They suggested that this connectivity might not only make the two sides dependent on each other but also violate the independence of the time and intensity pathways. Thus, the discharge of NM neurons may be affected by activities in the NA and NL of both sides. Because this hypothesis directly bears on the rationale of the present paper, we tested whether NM fibers receive regulatory input from the contralateral side via $\mathrm{SO}$.

We obtained relevant data from 6 of the 21 neurons under Ketamine and Diazepam anesthesia and 7 neurons without Diazepam. We took this precaution because Diazepam is known to potentiate GABA-mediated inhibition. The test procedures were as follows. For example, we obtained a rate-intensity curve for a neuron from the right NM by systematically increasing sound intensity in the right ear. We then applied an intensity above the asymptotic level on the left side while varying the right stimulus as before. Finally, we kept the right intensity constant at the asymptotic level while increasing the left stimulus systematically. Because NM neurons do not respond, all we get by these procedures is two curves, a flat curve for ipsilateral stimulation at a constant level and a sigmoid curve for stimulation of the ipsilateral side with increasing intensities (Fig. 10). Diazepam did not affect these relationships.

\section{DISCUSSION}

\section{Insensitivity of binaural discharge rate to IID}

One possible method to reduce the effects of IID on NL neurons is to equalize the afferent discharge rate between the two sides. NM receives bilateral GABAergic input from the superior olivary nucleus as mentioned above. We found that the impulse rates of NM fibers of the two sides varied independently of each other and increased monotonically with sound intensity. Thus, there is no mechanism available to equalize the impulse rates of the left and right afferent sources to nucleus laminaris, so the tolerance to IID must be established at the level of NL neurons.

The binaural discharge rate of NL neurons can be insensitive to IID under certain conditions. If the binaural discharge rate for the most favorable ITD were equal to the sum of the monaural rates, then it would not matter which side contributes the smaller or larger rate. However, the binaural discharge rate for the most
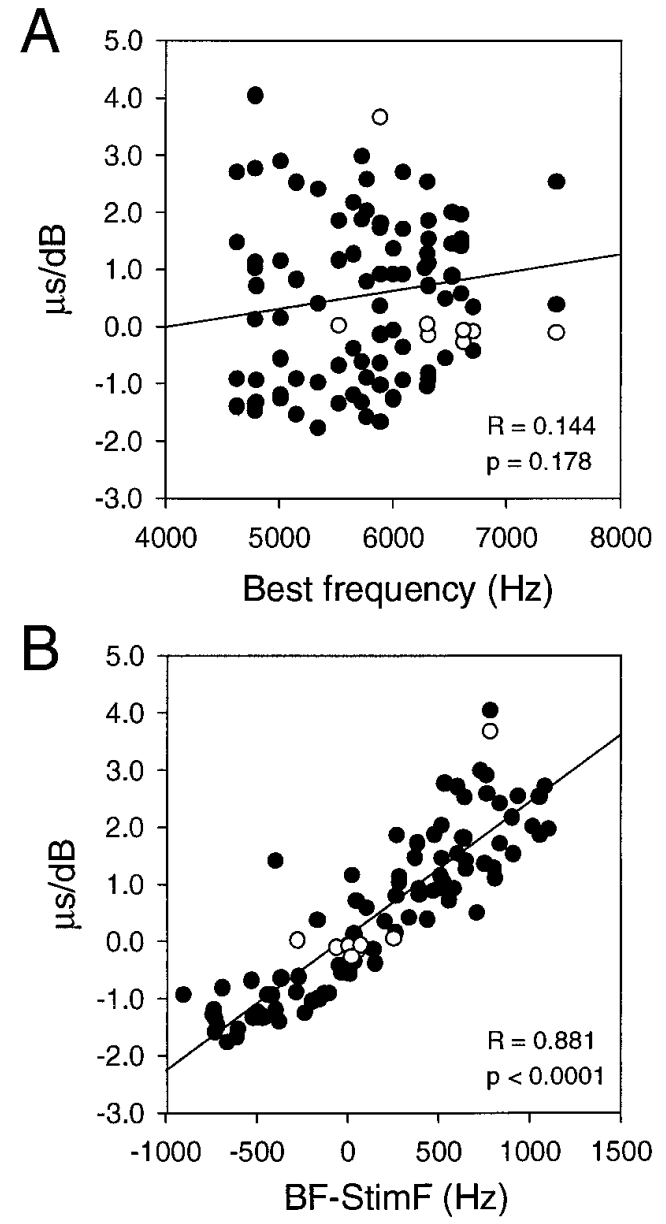

Figure 9. Summary of frequency-dependent shifts in mean phase (MP) in neurons of nucleus magnocellularis. Each point represents the slope of the MP versus the IID regression line obtained from each neuron. The slope values are not correlated with the neuron's BF $(A)$. There is a clear correlation between the slope values and the difference between $\mathrm{BF}$ and stimulating frequency $(B)$. Filled symbols indicate neurons that showed statistically significant regression coefficients $>0.8$ with $p<0.05$. Open symbols indicate neurons that did not meet these criteria.

favorable ITD is not equal to the sum of monaural discharge rates but, rather, to a value 1.5 times larger (Peña et al., 1996). This fact indicates that the binaural discharge rate is derived by multiplicative processes. If the multiplication of the ipsi- and contralateral probabilities of afferent impulses determines the probability of firing of NL neurons, IID should have no effect on the binaural discharge rate, because it does not matter which side has the higher or lower probability of discharge. When ABI is kept constant, IID increases the firing probability of one input by a certain amount and decreases that of the other input by the same amount. This possibility can occur when sound intensity changes within the monotonic range of afferent fibers.

\section{Effects of IID on the mean interaural phase}

The discharge rates of NL neurons but not their MIPs changed with ABI (Peña et al., 1996). In contrast, the discharge rate in response to tonal stimuli did not change with IID, but MIP did. These MIP shifts do not appear to be based on properties intrinsic to NL neurons but, rather, can be solely accounted for by changes in the timing of afferent impulses. The magnitude of MP shifts in NM neurons is similar to that of MIP shifts in NL neurons, and 
A

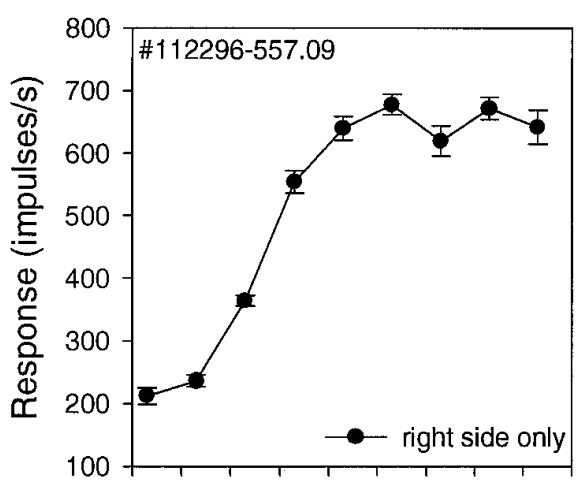

Figure 10. Rate-intensity curves of two magnocellular fibers for binaural and monaural stimulation. These data were obtained without Diazepam, because this drug potentiates GABA-mediated inhibition. $A, C$, Rate-intensity curves for monaural stimulation. $B, D$, For the same fibers shown in $A$ and $B$, sound intensity was kept constant at $55 \mathrm{~dB}$ SPL in one ear while it was progressively increased on the other side.

C
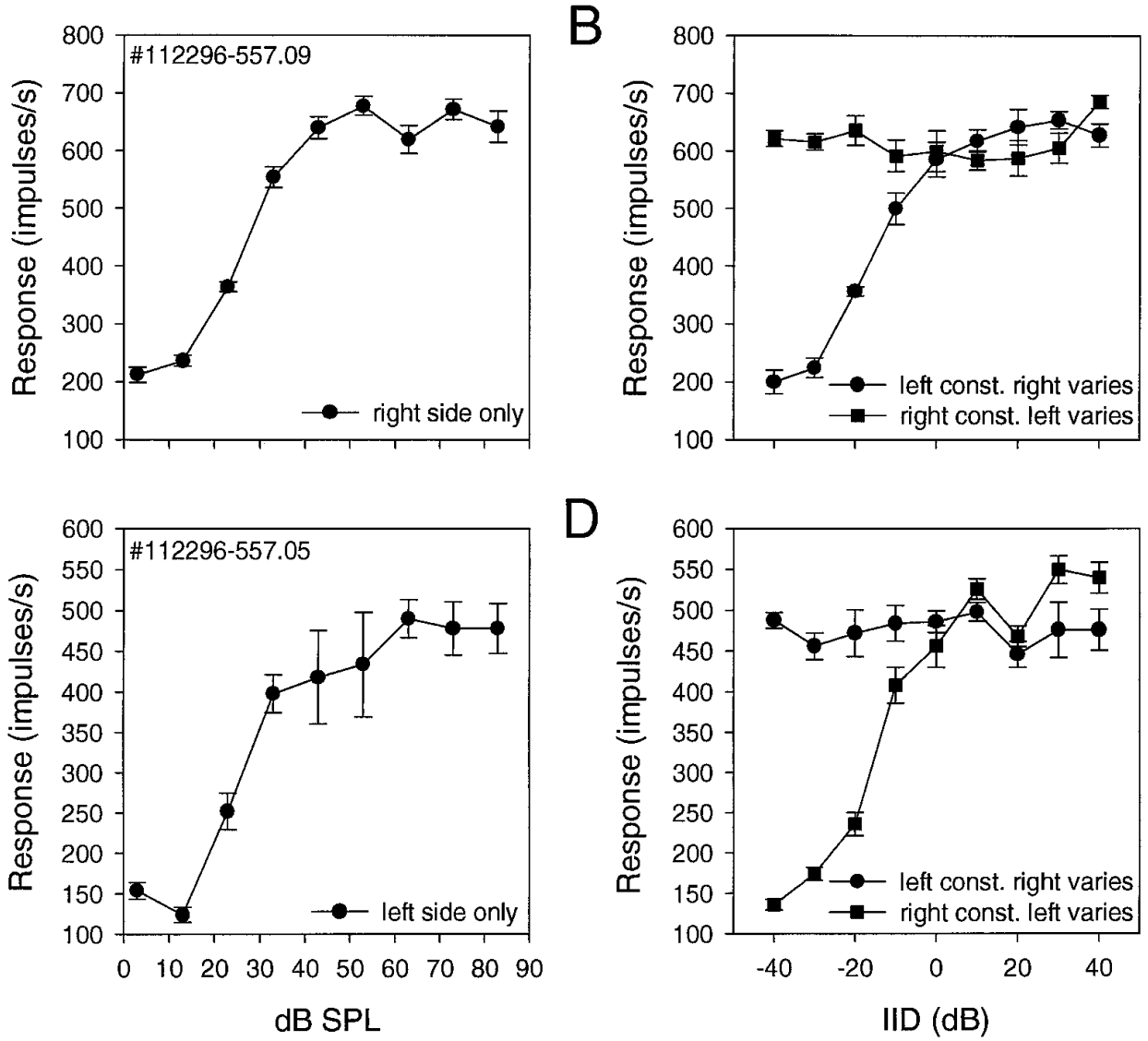

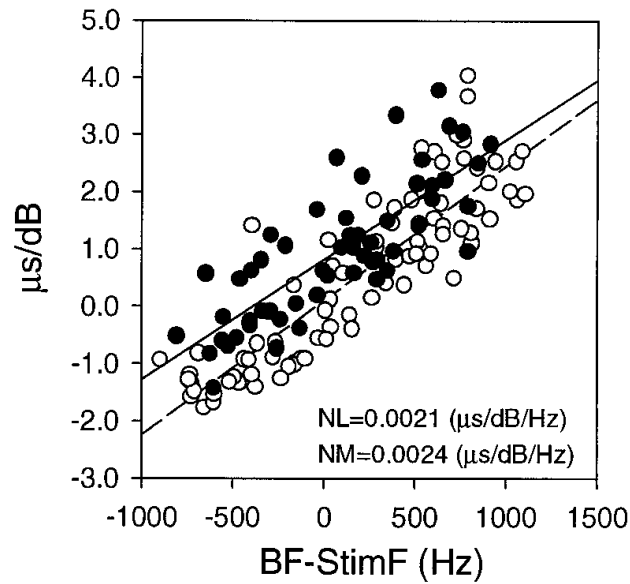

Figure 11. Comparison of frequency-dependent phase shifts between nucleus magnocellularis and nucleus laminaris. Figures $7 B$ and $9 B$ are superimposed on each other to show the similarity in the magnitude and direction of phase shifts caused by changes in frequency and intensity. Filled and unfilled circles denote NL and NM neurons, respectively. The linear regression coefficients for the two distributions are shown at the bottom.

the sign of MIP shifts can also be predicted from that of MP shifts in NM neurons (Fig. 11). These agreements can be explained as follows: NL neurons fire maximally when the sum of acoustic and neural delays for one side equals that of the opposite side; i.e., $A_{\mathrm{L}}$ $+N_{\mathrm{L}}=A_{\mathrm{R}}+N_{\mathrm{R}}$, where $A$ denotes acoustic delay and $N$ the sum of all neural delays that contribute to the delay of phase-locked impulses, and subscripts " $L$ " and " $R$ " indicate left and right. Thus, for example, a stimulus frequency below an NM neuron's BF causes an increase in the $N_{\mathrm{R}}$ of their target NL neuron. A larger $N_{\mathrm{R}}$ must be matched by a smaller $A_{\mathrm{R}}$ for a coincidence to occur in the same NL neuron. A decrease in $A_{\mathrm{R}}$ means a shift of the sound source toward the right ear in free field or a decrease in acoustic transmission time to the right ear in dichotic stimulation. Thus, MIP of this NL neuron should shift toward the right ear. This is exactly what happens in NL neurons when a frequency below their $\mathrm{BFs}$ and IIDs favoring the right ear are combined. MIP does not change if afferent neurons show no MP shifts with intensity as this condition was nearly realized with $\mathrm{BF}$ and broadband signals.

MP shifts in NM neurons are likely to come from the auditory nerve. In all animals studied so far, the MPs of primary auditory fibers are known to increase (lag) or decrease (lead) systematically with sound intensity when the stimulus frequency is below or above their BFs, respectively. Changes in sound intensity have little effects on MP when the stimulus frequency is equal to the fibers' BFs (squirrel monkeys: Andersen et al., 1971; starlings: Narins and Gleich, 1986; pigeons: Hill et al., 1989) (barn owls: C. Köppl, personal communication).

We observed differences in the magnitude of intensitydependent phase shifts between frequencies below and above BF. Such an asymmetry may have been seen by Andersen et al. (1971) when they stated, "Above BF, an opposite tendency often prevails if a change in phase angle occurs" (p. 1131). This difference between frequencies below and above BF may be attributable to asymmetrical expansion of frequency tuning curves with intensity, as seen in some of the data figures in their paper. The lowfrequency side of BF appears to expand more than the highfrequency side. This explanation would not apply to barn owls, 
because the tuning curves of barn owls' primary auditory neurons do not expand asymmetrically (C. Köppl, personal communication). At any rate, this type of phase shift in the auditory nerve and higher-order neurons is likely to originate in the inner ear, because phase shifts of similar nature occur in inner hair cell potentials and basilar membrane mechanics (Rhode and Robles, 1974; Sellick et al., 1982; Dallos, 1986; Cooper and Rhode, 1992; Rhode and Cooper; 1996).

It should be pointed out that the type of temporal shifts described in the present paper is different from the changes of first-impulse latencies. The former is in the microsecond range, dependent on frequency, and does not obey the rule of time and intensity trade, whereas the latter is in the millisecond range, independent of frequency, and shows time and intensity trade in which the latency becomes smaller as sound intensity increases. Similar comparisons have been made in other studies (see, for example, Yin and Kuwada, 1983b; Volman and Konishi, 1990).

\section{Other studies}

There are few other studies that address the effects of IID on ITD processing at the level of coincidence detection. Studies of MSO neurons show both similarities and differences to the present results. Goldberg and Brown (1969), using the neuron's BF, did not find any effects of IID on the MIP in the dog's MSO. Crow et al. (1978) reported for the kangaroo rat's superior olivary nucleus an average MIP shift of $8 \mu \mathrm{sec} / \mathrm{dB}$, which was obtained by lumping shifts in both directions. Yin and Chan (1990) reported small shifts in the MIP as a function of IID in the cat's MSO, in which the average rate of change for 3 neurons tested was 0.0016 cycles/dB.

Relatively few systematic studies of the effects of IID on higherorder ITD-sensitive neurons have been carried out, with notable exceptions of those by Yin and Kuwada (1983a,b), who found shifts in MIP for some neurons of the cat's inferior colliculus. They reported that the effect was independent of the stimulus frequency and that there was a continuous distribution of cells from those with essentially no sensitivity to intensity to others with high sensitivity, with a mean shift of $5.82 \mu \mathrm{sec} / \mathrm{dB}$. Olsen et al. (1989) found systematic shifts of the most favorable ITD with changes in IID in some neurons of the optic tectum of the barn owl. Changes of IID by $15.4 \pm 6.6 \mathrm{~dB}$ (half-height width of IID tuning curves) caused shifts in the favorable ITD ranging from 0 to $27 \mu \mathrm{sec}$. These shifts in ITD were always favoring the same ear in which intensity was increased. These shifts $/ \mathrm{dB}$ are similar to those we obtained for broadband noise stimulation.

At the behavioral level, IIDs appear to make only small contributions to azimuthal localization in barn owls. Moiseff (1989b) used earphones for independent control of ITD and IID to study the localization of phantom sources in two barn owls. When ITD was kept at zero and IID was varied, the owls tended to miss $0^{\circ}$ azimuth by $0.55^{\circ} \mathrm{dB}$ IID on the side of louder sound. This observation is consistent with our neurophysiological results with broadband noise signals in which the MIP shifted toward the louder ear by $0.71 \pm 0.22 \mu \mathrm{sec} / \mathrm{dB}$ IID. Because $0.55^{\circ}$ in azimuth corresponds to $\sim 2.2 \mu \mathrm{sec}$ in ITD (Moiseff, 1989b), the behavioral and neural shifts are not radically different from each other, particularly when the large variances associated with these measurements are considered.

\section{The independence of ITD and IID}

One of the original reasons for the view that ITD and IID are independently processed was the physiological differences be- tween NM and NA (Sullivan and Konishi, 1984a). Aside from the presence and absence of phase locking in the NM and NA, respectively, NM neurons were thought to have a smaller average dynamic range $(17 \mathrm{~dB})$ than NA neurons $(30 \mathrm{~dB})$. However, a recent study does not find such a difference, although because of their low spontaneous rates NA neurons show a greater range of discharge rates than do NM neurons (Peña et al., 1996) (C. Köppl, personal communication). We have learned in the present study that the processing of ITD is relatively independent of IID, because it uses phase-locked impulses the timing of which is relatively insensitive to variation in intensity when the stimulus is a broadband signal. The opposing phase shifts caused by spectral components above and below BF of a noise stimulus apparently cancel each other out. The mechanisms for this process must reside in the inner ear.

\section{REFERENCES}

Anderson DJ, Rose JE, Hind JE, Brugge JF (1971) Temporal position of discharge in single auditory nerve fibers within the cycle of a sine-wave stimulus: frequency and intensity effects. J Acoust Soc Am 49:1131-1139.

Batschelet E (1981) Circular statistics for biology. London: Academic.

Brainard MS, Knudsen EI, Esterly SD (1992) Neural derivation of sound source location: resolution of spatial ambiguities in binaural cues. J Acoust Soc Am 91:1015-1027.

Carr CE (1993) Delay-line models of sound localization in the barn owl. Am Zool 33:79-85.

Carr CE, Boudreau RE (1993) Organization of the nucleus magnocellularis and the nucleus laminaris in the barn owl: encoding and measuring interaural time differences. J Comp Neurol 334:337-355.

Carr CE, Konishi M (1990) A circuit for detection of interaural time differences in the brain stem of the barn owl. J Neurosci 10:3227-3246.

Cooper NP, Rhode WS (1992) Basilar membrane mechanics in the hook region of cat and guinea-pig cochleae: sharp tuning and nonlinearity in the absence of baseline shifts. Hear Res 63:163-190.

Crow G, Rupert AL, Moushegian G (1978) Phase locking in monaural and binaural medullary neurons: implication for binaural phenomena. J Acoust Soc Am 64:497-501.

Dallos P (1986) Neurobiology of the cochlear inner and outer hair cells: intracellular recordings. Hear Res 22:185-198.

Goldberg JM, Brown PB (1969) Response of binaural neurons of dog superior olivary complex to dichotic tonal stimuli: some physiological mechanisms of sound localization. J Neurophysiol 32:613-636.

Heiligenberg WF (1991) Neural nets in electric fish. Cambridge: MIT.

Hill KG, Stange G, Mo J (1989) Temporal synchronization in the primary auditory response in the pigeon. Hear Res 39:63-74.

Jhaveri S, Morest DK (1982) Neuronal architecture in nucleus magnocellularis of the chicken auditory system with observations on nucleus laminaris: a light and electron microscope study. Neuroscience 7:809-836.

Joseph AW, Hyson RL (1993) Coincidence detection by binaural neurons in the chick brain-stem. J Neurophysiol 69:1197-1211.

Konishi M, Sullivan WE, Takahashi T (1985) The owl's cochlear nuclei process different sound localization cues. J Acoust Soc Am 78:360-364.

Lachica EA, Rübsamen R, Rubel EW (1994) GABAergic terminals in nucleus magnocellularis and laminaris originate from the superior olivary nucleus. J Comp Neurol 348:403-418.

Maunsell JHR, Nealey TA, Depriest DD (1990) Magnocellular and parvocellular contributions to responses in the middle temporal visual area (mt) of the macaque monkey. J Neurosci 10:3323-3334.

Merigan WH, Maunsell JHR (1993) How parallel are the primate visual pathways? Annu Rev Neurosci 16:369-402.

Moiseff A (1989a) Binaural disparity cues available to the barn owl for sound localization. J Comp Physiol [A] 164:629-636.

Moiseff A (1989b) Bi-coordinate sound localization by the barn owl. J Comp Neurol 164:637-644.

Narins P, Gleich O (1986) Phase response of low frequency cochlear ganglion cells in the starling. In: Auditory frequency selectivity (Moor BCJ, Patterson RD, eds), pp 209-216. New York: Plenum.

Olsen JF, Knudsen EI, Esterly SD (1989) Neural maps of interaural time and intensity differences in the optic tectum of the barn owl. J Neurosci 9:2591-2605. 
Overholt EM, Rubel EW, Hyson RL (1992) A circuit for coding interaural time differences in the chick brainstem. J Neurosci 12:1698-1708.

Parks TN (1981) Morphology of axosomatic endings in the avian cochlear nucleus: nucleus magnocellularis of the chicken. J Comp Neurol 203:425-440.

Peña JL, Viete S, Albeck Y, Konishi M (1996) Tolerance to sound intensity of binaural coincidence detection in the nucleus laminaris of the barn owl. J Neurosci 16:7046-7054.

Press WH, Flannery BP, Teutolsky SA, Vetterling BP (1988) Numerical recipes in C: the art of scientific computing. Cambridge: Cambridge UP.

Rhode WS, Cooper NP (1996) Nonlinear mechanics in the apical turn of the chinchilla cochlea in vivo. Auditory Neurosci 3:101-121.

Rhode WS, Robles L (1972) Evidence from Mössbauer experiments for nonlinear vibration in the cochlea. J Acoust Soc Am 80:588-596.

Sachs MB, Sinnott JM (1978) Responses to tones of single cells in nucleus magnocellularis and nucleus angularis of the redwing blackbird (Agelaius phoeniceus). J Comp Physiol [A] 126:347-361.

Sellick PM, Patuzzi R, Johnstone BM (1982) Measurement of basilar membrane motion in the guinea pig using the Mössbauer technique. J Acoust Soc Am 72:131-141.

Sullivan WE, Konishi M (1984a) Segregation of stimulus phase and intensity coding in the cochlear nucleus of the barn owl. J Neurosci 4:1787-1799.

Sullivan WE, Konishi M (1984b) Neural map of interaural phase difference in the owl's brainstem. Proc Natl Acad Sci USA 83:8400-8404.
Takahashi T, Konishi M (1988a) The projections of the nucleus angularis and the nucleus laminaris to the lateral lemniscal nuclear complex of the barn owl. J Comp Neurol 274:212-238.

Takahashi T, Konishi M (1988b) Projections of the cochlear nucleus and the nucleus laminaris to the inferior colliculus of the barn owl. J Comp Neurol 274:190-211.

Takahashi T, Moiseff A, Konishi M (1984) Time and intensity cues are processed independently in the auditory system of the owl. J Neurosci 4:1781-1786.

Volman SF, Konishi M (1990) Comparative physiology of sound localization in four species of owls. Brain Behav Evol 36:196-215.

Warchol ME, Dallos P (1990) Neural coding in the chick cochlear nucleus. J Comp Physiol [A] 166:721-734.

Yin TCT, Chan JCK (1990) Interaural time sensitivity in the medial superior olive of the cat. J Neurophysiol 64:465-488.

Yin TCT, Kuwada S (1983a) Binaural interaction in low-frequency neurons in inferior colliculus of the cat. I. Effects of long interaural delays, intensity, and repetition rate on interaural delay function. J Neurophysiol 64:981-999.

Yin TCT, Kuwada S (1983b) Binaural interaction in low-frequency neurons in inferior colliculus of the cat. II. Effects of changing rate and direction of interaural phase. J Neurophysiol 50:1000-1019.

Young SR, Rubel EW (1986) Embryogenesis of arborization pattern and topography of individual axons in n. laminaris of the chicken brain-stem. J Comp Neurol 254:425-459. 